\title{
Geometric Alignment Of Cone-Beam Helical-Trajectory Micro-Tomography Data Using a PI-Line Difference Metric
}

\author{
Olaf Delgado-Friedrichs ${ }^{1,2}$, Andrew M. Kingston ${ }^{1,2, *}$, Shane J. Latham ${ }^{1,2}$, Glenn R. Myers ${ }^{1,2}$ and Adrian P. \\ Sheppard ${ }^{1,2}$. \\ 1. Dept. of Applied Mathematics, Research School of Physics and Engineering, The Australian National \\ University, Canberra, ACT 2601, Australia. \\ 2. CTLab: National Laboratory for Micro Computed-Tomography, Advanced Imaging Precinct, The \\ Australian National University, Canberra, ACT 2601, Australia. \\ * Corresponding author, andrew.kingston@anu.edu.au.
}

Software methods for post-acquisition alignment of projection data in micro- and nano- computedtomography (CT) have been applied successfully in recent years, in most cases eliminating the need for precise geometric alignment of instrument components. One strategy is to search for alignment parameter values that maximize the sharpness in a filtered back-projection reconstruction [1]. A potentially much faster and more robust alternative is based on the projection differences in opposing rays along PI-lines. This has been applied to both circular and helical scanning trajectories with mixed results [2]. Here we consider their use with double-helix [3] as well as space-filling trajectories [4].

We assume a lens-less fine-focus geometry where magnification is achieved through the expanding spherical wave-front of X-rays emitted from a micro(nano)-focus X-ray source $(S)$ that is a distance $S_{l}$ from the rotation axis. A flat-panel detector $(D)$ is located a distance $D_{l}$ from the rotation axis (in the opposite direction). The relative orientation of the system $S-D$ with respect to the specimen coordinate system is specified by an elevation $z$ and a horizontal angle $\theta$. A generalized helical trajectory in this setting is then any sequence $\left(\theta_{i}, z_{i}\right)=(i \cdot \Delta \theta, i \cdot \Delta z), i=1, \ldots, \Omega$, where $\Omega$ is the total number of projections, $\Delta \theta$ giving the angular translation between source points, and $\Delta z$ giving the vertical translation.

A double-helix trajectory uses less radiographs per revolution than a helix, but incorporates an additional set of helical data with all angles offset by half a revolution. For details on the definition, properties and advantages of the DHT, we refer to [3]. A space-filling trajectory can be defined simply as a low-pitch helix with a large stride, in which the horizontally scaled pairs $\left(i \cdot S_{l} \cdot \Delta \theta, i \cdot \Delta z\right)$ form an approximately hexagonal pattern. For details on the definition, properties and advantages of the SFT, we refer to [4].

The center position of the source and detector at the $i$-th radiographic measurement are found as $s_{i}=(-$ $\left.S_{l} \cdot \cos \left(\theta_{i}\right),-S_{l} \cdot \sin \left(\theta_{i}\right), z_{i}\right)$ and $d_{i}=\left(D_{l} \cdot \cos \left(\theta_{i}\right), D_{l} \cdot \sin \left(\theta_{i}\right), z_{i}\right)$, respectively. It is straightforward to compute the straight line (known as a PI-line) between $s_{i}$ and $s_{j}$ for any index pair $i, j$, and thus obtain the detector value $r_{i, j}$ (if it exists) corresponding to the ray from $s_{i}$ going through $s_{j}$ for the $i$-th radiograph. Pairs of source/detector positions that are too close in $\theta$ or too far in $z$ lead to PI-lines that do not intersect the detector, in which case the associated value $r_{i, j}$ will be undefined.

We define two matrices, $M=\left(m_{i, j}\right)$ and $A=\left(a_{i, j}\right)$ for $i, j=1, \ldots, \Omega$. Set $m_{i, j}=1$ if both $r_{i, j}$ and $r_{j, i}$ are defined, $m_{i, j}=0$ otherwise. Elements of $A$ are then found as $a_{i, j}=m_{i, j} \cdot r_{i, j}$. The accumulated ray difference along PI-lines is computed as $e=\left|A-A^{T}\right| /|M|$ for an appropriate matrix norm |.| (e.g. the $\mathrm{L}^{2}$ norm). Repeating the computation based on a hypothetical misalignment of the system $S$ - $D$, we derive an analogous value $e(\boldsymbol{p})$ for $\boldsymbol{p}$ an appropriate parameter vector describing misalignment. Our task is to find a vector $\boldsymbol{p}$ out of the set $\boldsymbol{P}$ of potential misalignments that minimizes $e$; it is assumed that $\boldsymbol{p}_{\min }$ best 
describes the current system geometry. Note that $e$ can be computed in time $O\left(\Omega^{2}\right)$. Here we optimized $e$ via a series of constant-step 1-parameter scans followed by an application of Powell's method.

Figure 1 shows the uncorrected and corrected reconstructions for a set of 1950 radiographs with simulated misalignments on a space-filling trajectory, generated from a digital phantom of size $512 \mathrm{x}$ $512 \times 1024$ voxels with a voxel size of $3.2 \mu \mathrm{m}$. The geometry was such that $S_{l}=1.6384 \mathrm{~mm}$ and $D_{l}=$ $80.2816 \mathrm{~mm}$, corresponding to a magnification factor of 50 and a detector pixel size of $0.16 \mathrm{~mm}$. The simulated detector size was 592 pixels along each axis, resulting in a cone angle of $60^{\circ}$. The total vertical extent was $5.4012 \mathrm{~mm}$, with $\Delta z=2.771 \mu \mathrm{m}$ and $\Delta \theta=6.36^{\circ}$. The estimated misalignments matched the known values within fractions of an optimal unit (OU) [1].

Figure 2 shows the uncorrected and corrected reconstructions for a cylindrical sample of white beech wood, 3mm in diameter, acquired with a double-helix trajectory using a total of 14136 projections (7068 per helix). The data was taken with $S_{l}=3 \mathrm{~mm}$ and $D_{l}=312.5 \mathrm{~mm}, \Delta z=1.344 \mu \mathrm{m}$ and $\Delta \theta=0.125^{\circ}$. A 3040 x 3040 pixel detector with a pixel size of $0.139 \mathrm{~mm}$ was used. The effective cone angle was again $60^{\circ}$, and the magnification factor was 105.2. The estimated misalignments using the PI-line difference and the sharpness metric differed by less than an OU in all parameters, in most cases only by a fraction of an OU. We note that the PI-line difference metric was significantly more sensitive to intensity fluctuations in this case than sharpness, so that a successful alignment required an additional effort in correcting these.

We conclude that the PI-line difference metric is an equally robust and significantly faster alternative to sharpness when used on SFT data, and typically robust enough for DHT data with a potential need for additional preprocessing [5].

\section{References:}

[1] A. Kingston et al, Medical Physics 38 (2011) p. 4934.

[2] A. Kingston et al, Proceedings ICTMS (2013) p. 101.

[3] T. Varslot et al, SPIE Optical Engineering + Applications (2012), p. 850614.

[4] A. M. Kingston et al, SPIE Optics and Photonics (2016) p. 9967-38.

[5] The authors acknowledge the financial support of the Australian Research Council and FEI-Thermo Fisher Scientific through Linkage Project LP150101040, and the use of super-computer time provided by Australia's National Computational Infrastructure (NCI).

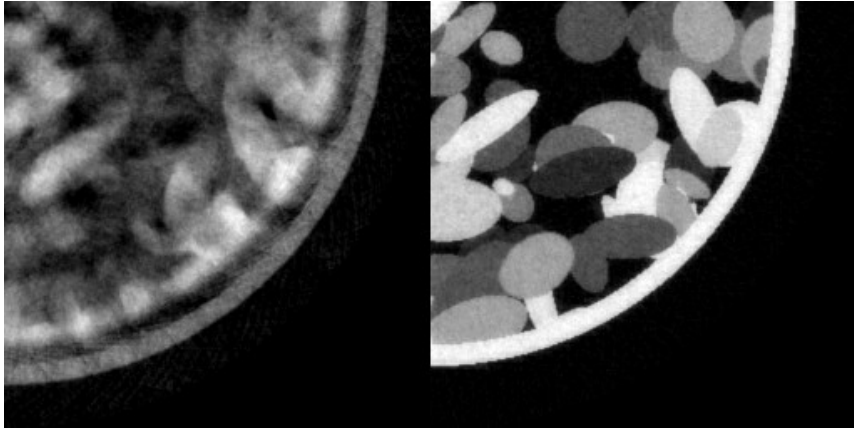

Figure 1. Reconstructions of an SFT dataset from a digital phantom with simulated misalignments. Left: uncorrected. Right: corrected for estimated misalignments using the PI-line difference metric.

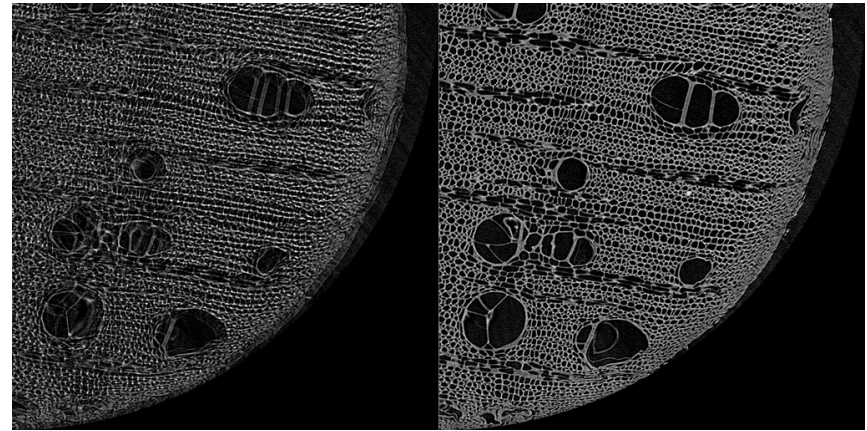

Figure 2. Reconstructions of a white beech DHT dataset. Left: uncorrected. Right: corrected for estimated misalignments using the PI-line difference metric. 